\title{
Immediate effect of exoskeleton hbp (human body posturizer) in healthy individuals:
}

\section{A pilot study.}

Rosa Grazia Bellomo ${ }_{\mathrm{ABCDEF}}$, Giovanni Barassi ${ }_{\mathrm{ABCDEF}}$, Simona Maria Carmignano ${ }_{\mathrm{ABCDEF}}$,

Giacomo Melle $^{3}{ }_{\mathrm{ABCDEF}}$ Emilio Ancona ${ }_{\mathrm{ABCDEF}}$, Daniele Porto $^{3}{ }_{\mathrm{ABCDEF}}$ Ilaria Pecoraro ${ }_{\mathrm{ABCDEF}}$, Raoul Saggini ${ }_{\text {ABCDEF }}^{4}$

${ }^{1}$ School of Specialties in PRM, Department of Medicine and Science of Aging, "G.d'Annunzio" University, Chieti,Italy

${ }^{2}$ Faculty of Physiotherapy "G.d'Annunzio" University-Chieti

${ }^{3}$ School of Specialties in PRM, "G.d'Annunzio" University, Chieti, Italy

${ }^{4}$ School of Specialties in PRM, "G. d'Annunzio" University, Chieti, Italy Department of Medical Sciences, Oral and Biotechnology,

"G.d'Annunzio" University, Chieti, Italy

\begin{abstract}
Without the intervention of muscular strength and proprioceptive function, humans would not be able to maintain the upright position, it would tend to fall. This continuous solution of instability promotes a more dynamicity, because an unstable balance structure can be put in motion more easily than a stable balanced structure. In fact, human body is capable more of mobility at the expense of stability. Postural control is the ability of the body to manage high instability situation, near to the point where postural balance is no longer recoverable. It is based on coordinated and synergistic mechanisms of proprioceptive and exteroceptive act. The new exoskeleton HBP (Human Body Posturizer), has the objective of physiologically aligning the column to get a beneficial effect on the alignment of the global subject by stimulating peripheral proprioceptors. We have developed an evaluation protocol through the use of measurement instruments: the Biometrics digitized system and the I- Moove Coordination Test, in a sample of 50 healthy young subjects, randomly taken from healthy population, to detect changes in postural parameters and motor coordination ,before, after and during use of HBP. Results show that the exoskeleton immediately changes the posture and balance of the subjects examined, surely acting also on peripheral receptors toward a physiological spinal alignment.
\end{abstract}

Key words: posture, balance, exoskeleton

\section{Introduction}

Without the intervention of muscular strength and proprioceptive function, humans would not be able to maintain the upright position, it would tend to fall $[1,2]$. This continuous solution of instability promotes a more dynamicity, because an unstable balance structure can be put in motion more easily than a stable balanced structure [3]. In fact, human body is capable more of mobility at the expense of stability $[4,5]$.

Postural control is the ability of the body to manage high instability situation, near to the point where postural balance is no longer recoverable. It is based on coordinated and synergistic mechanisms of proprioceptive and exteroceptive act $[6,7]$.

Balance is the property of a body to maintain the same position in a well defined spatial orientation and it is in relationship to the inertial forces acting on the body and the inertial characteristics of the segments of the body [8]. Kandel charts and stands the balance in: static, dynamic, stable, unstable and indifferent balance $[9,10,11,12]$.
The posture management in CNS involves the presence of interactions between external forces, such as gravity, and internal forces, such as the neuromuscular forces $[13,14,15]$.

In modern rehabilitation there are many ways to improve and rehabilitate posture [16,17].

The development of powerful, efficient, adaptable, lightweight and low-cost mechanisms is imperative in achieving applicable exoskeletons and fulfilling the goal of integration of robots into our daily lives. On the other hand, equally important are the control methods that provide the necessary interface between the human user and robotic mechanism. The control framework should be able to predict the human intentions and apply them to the mechanism at the correct time to achieve the desired human-robot cooperation and co-adaptation[20].

The new exoskeleton HBP (Human Body Posturizer), has the objective of physiologically aligning the column to get a beneficial effect on the alignment of the global subject by stimulating peripheral proprioceptors $[18,19]$. Thanks to its versatility, HBP can be used in all age groups, from 
the youngest to the elderly; also HBP can be worn as a whole or separately. It can be also used in aquatic environment. It does not aggravates the patient because its overall weight is around $1.5 \mathrm{~kg}$. Modern studies show that wearing HBP since thirty to forty minutes a day, will cause a postural reorganization, a loads redistribution and a pains disappearance; then the subject performs a proper exercise both in a health condition, and in a disease condition. Therefore, it is possible to use HBP not only as a cure but also as essential prevention and aid in the correctly performance of ADL to achieve an improvement in quality of life [19].

It is used in orthopedic disorders (scoliosis, dysmorphic column, after-effects of trauma, radicular pathologies) and neurological one (SM, parkinsonism, ataxia improving the ' balance and stability, reducing the risk of falls of $80 \%$. It acts directly on frontal lobes and prerolandic region, which are important for the movement; using this instrument, the subject trains itself to increase the capability to perform a specific movement through the stimulation of the area mentioned before [18]. We have developed a research project, in order to assess whether the use of the device exoskeleton HBP could, with immediate effect, be beneficial on overall alignment of the subject and on the static, dynamic performance and during specific examinations about proprioception and balance. We have developed an evaluation protocol through the use of measurement instruments:

the Biometrics digitized system and the I- Moove Coordination Test, in a sample of 50 healthy young subjects, randomly taken from healthy population, to detect changes in postural parameters and motor coordination ,before, after and during use of HBP.

\section{Material and methods}

This experimental study and the research protocol is compatible with the Declaration of Helsinki. This study was conducted at the University Centre of Physical and Rehabilitation Medicine, „Gabriele D’Annunzio" University in Chieti.

The Human Body Posturizer (HBP) is a totally articulated orthotic (exoskeleton - suit Hi-Tech) developed to bring the subject in a posture as possible physiological. The HBP mechanics is fully mobile (differs from other orthotic fixed that prevent the movement of the underlying structure) and allow active movement of the muscular structures .

HBP interacts with joints, allows the patient to change the posture and maintain balance, both static and dynamic, through peripheral receptors. Moreover, being constituted by several segments which are modelled on the body, it acts particularly on peripheral receptors (skin and muscle and fascia mechanoreceptors) that by sending pulses to the supra-axial nerve centers, will allow the subject a new and better postural alignment. HBP is a totally articulated orthosis, made up of four basic elements that come into contact with various anatomic regions ; it is able to adapt to the physical characteristics of each individual leaving freedom of movement and allowing a continuous central reprogramming of the posture. (fig 1)

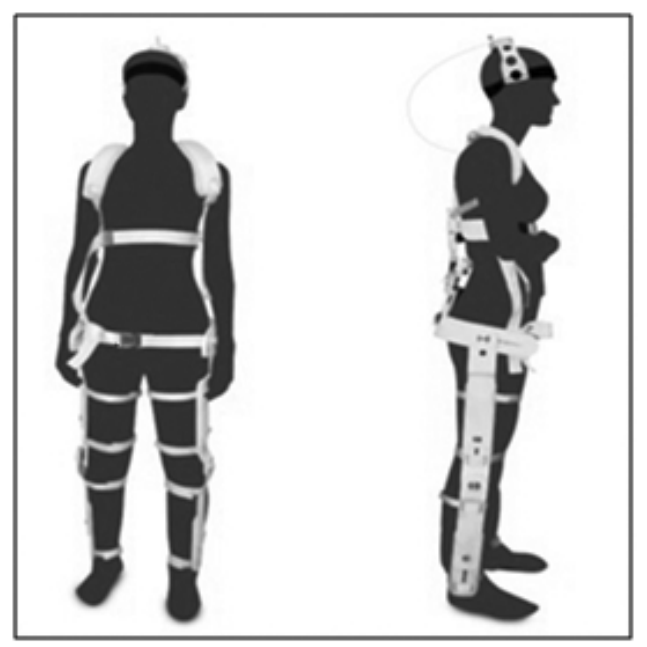

Figure 1 Human Body Posturizer (HBP)

For the study it was enrolled an homogeneous sample of 50 male and 50 female subjects from students of Faculty of Physiotherapy "G.d'Annunzio" University-Chieti

The sampling time was approximately 30 days.

The inclusion criteria of the study were:

- Age between 18 and 35

- Height between $155 \mathrm{~cm}$ and $200 \mathrm{~cm}$

- Weight less than $120 \mathrm{~kg}$

- Absence of systemic or neurological diseases

- No motor deficits, no trauma, no wounds

- No pain

- No foot-ankle dysmorphic subsystem.

To avoid learning and adaptation, we randomized the sample in two groups: Group A and Group B.

Group A followed this procedure:

1. Digitized Biometrics Evaluation without use of HBP (in figures "normal")

2. Check-up by I-moove Evaluation without use of HBP (in figures "normal")

3. Digitized Biometrics Evaluation with HBP

4. Check-up by I-moove Evaluation with HBP Group B followed the opposite process: 
1. Digitized Biometrics Evaluation wearing HBP

2. Check-up I-moove Evaluation wearing HBP

3. Digitized Biometrics Evaluation without HBP (in figures "normal")

4. Check-up by I-moove Evaluation without HBP (in figures "normal")

\section{Proprioceptive platform I-moove}

Imoove is a "sensory-motor" device for proprioceptive enhancing of trunk and lower limbs. Based on a Helispheric motion, it stimulates the vertebral natural movement because it allows to reproduce the spiral movements of muscle chains that make up the body system. Thanks to the instability of helispheric training, body increase postural and muscular control, essential for dynamic activity, and stimulate deep muscle proprioception and restore a better balance.

Another feature of the proprioceptive Imoove system is to be able to assess the stability, dynamic posture and coordination using the check-up program thanks to the presence of a monitor.(fig 2).

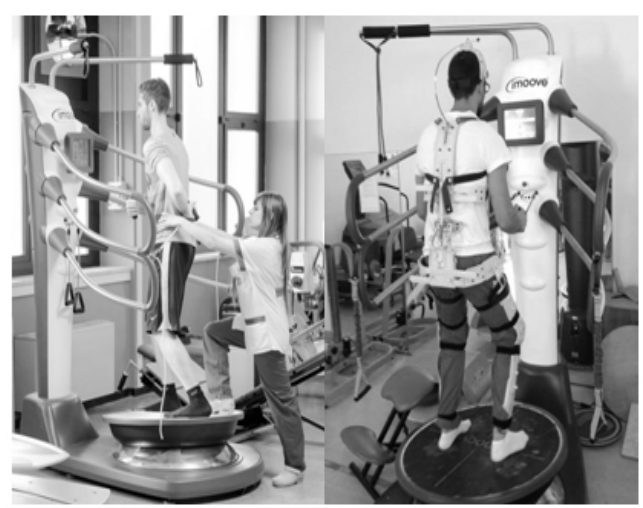

Figure 2 Proprioceptive platform I-moove

\section{Digitized Biometrics Evaluation}

The Digitalized Biometry is an instrumental evaluation that analyzes the information of the plantar surface (baropodometric exam) and balance (stabilometric exam). (fig 3)

The system used is the "Walk", made MultiSensor version; It consists of a $160 \mathrm{~cm}$ long platform with 25,600 active sensors and walkway of $160 \mathrm{~cm}$. This system is specific for the baropodometric and stabilometric analysis and for the complete analysis of the gait (minimum 3 rests on the platform).

The baropodometry is the examination of the plantar support $($ To $=$ pressure; Podo $=$ foot; metro $=$ measure); it is carried out with the Modular Electronic baropodometric system, consisting of a detection platform with thousands of active sensors interfaced to an acquisition software.

The resulting data will focus on the support surface and on the percentage of load in the static of both feet.

Stabilometry therefore has the objective of evaluating the postural control ability of a subject, in the standing position, through the quantification of postural sway, and the relative

contribution of various components of the postural system (visual, proprioceptive of different origin, labyrinthine). [21]

The parameters that are measured are: the ellipse surface of confidence, which contains $90 \%$ of sampled points and expresses the precision of the postural system; the Status-kinesigram (STKG), which represents the actual displacement of the COP (center of pressure) in reference to the support foot (ball-sway area-); the length (L), in millimeters, which represents the overall path of the COP (center of pressure) on the platform and is an index of energy expenditure by the system.

Gait analysis is performed by inviting the subject to walk spontaneously on the platform for about 35 seconds. This survey is repeated several times to identify the functional synergies during movement and to detect any gait and balance deficits. The parameters to consider are: bipodalic variation in the loading and distribution area.

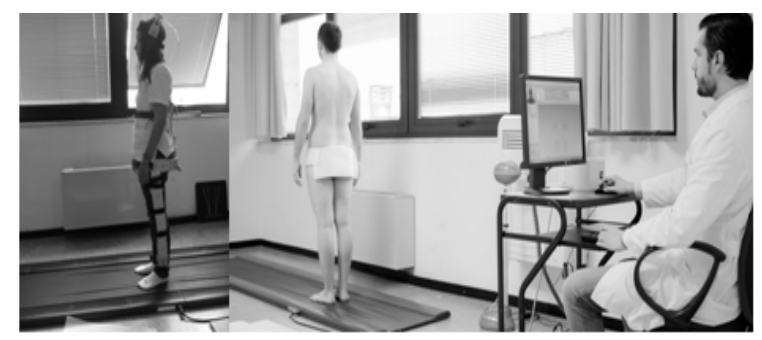

Figure 2 Proprioceptive platform I-moove

\section{Experimental Protocol}

All subjects were submitted to a postural assessment, including a three-dimensional visual analysis of the spine to look for any asymmetries and postural abnormalities visible and quantifiable (hypo-hyper lordosis, kyphosis, forward head posture, scoliosis 
Regardless of whether or not the HBP device, digitized biometrics consisted of the following procedural sequence:

- Baropodometry static: The patient is made to stand on the platform in a natural and relaxed position (stationary for 5-10 seconds), to evaluate the basic posture, the characteristics of the plantar surface, the difference percentage of the bearing and load surface between the two sides;

- Stabilometric Analysis: it was performed with parallel feet, 30 seconds with open eyes and 30 seconds with closed eyes; the anteroposterior and lateral-lateral oscillations and the area of the confidence ellipse area and the sway area have been taken into consideration for this study.

Gait analysis: the subject was walking on the platform forward and back for about 30 seconds in the most natural way possible. We evaluated the difference percentage of the plantar support surface and of the load between the two feet.

After this evaluation the patient with or without wearing HBP (it depends on the group in which patient was encloed) on to perform the check-up I-Moove. For this purpose, i-moove has been calibrated under these conditions: intensity level 2, sensitivity level 1; the duration of the check-up was 60 seconds. The data used for the study were: coordination index and stability index.

\section{Results}

The difference in the bearing surface in percent during gait analysis records an improvement in both groups, the group A passes from a difference of $7.86 \%$ (SD 6.81) to $5.78 \%$ (SD 4.37), the group $B$ passes by a difference equal to $6.43 \%$ (SD 5.18) to a difference wearing exoskeleton equal to $4.76 \%$ (SD 3.98), with a decrease of the average values of the percentage difference of the plantar surface support between left and right in the gait analisys with the use of HBP (fig 4).

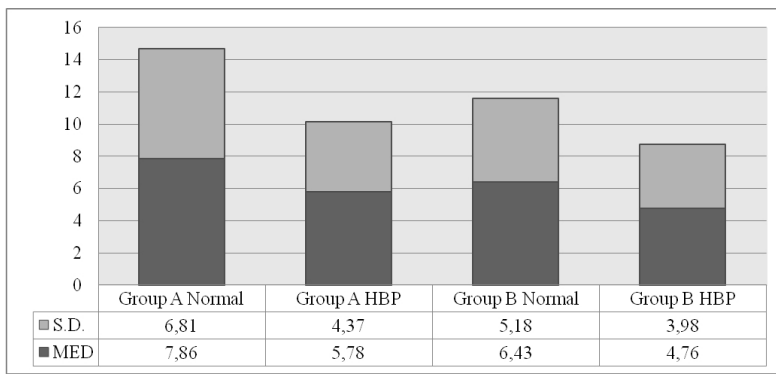

Figure 4 The difference in the bearing surface in percent during gait analysis
The difference of distribution of load in percent in gait analysis records an improvement in both groups, the group A passes by a difference of $10.46 \%$ (SD 13,24) to $8.23 \%$ (SD 7.59), the group B passes by a difference equal to $8.63 \%$ (SD 6.12) to a difference wearing exoskeleton equal to $7.06 \%$ (SD 5.05 ), with a decrease of the average values of the difference of the distribution of load percentage between left and right with the use of HBP (fig 5).

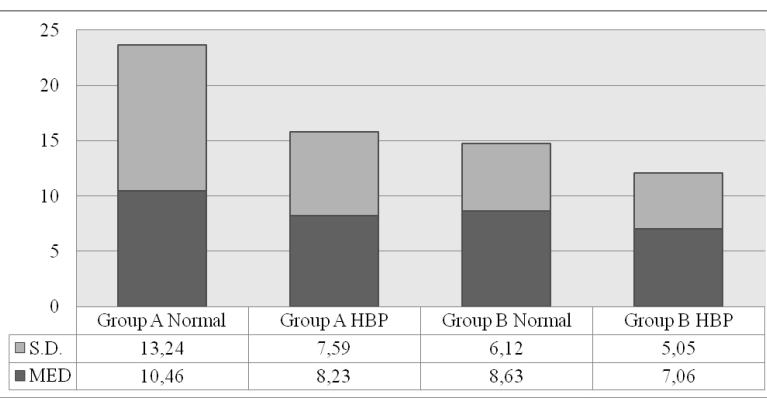

Figure 5 The difference of distribution of load in percent in gait analysis

The difference about the sway area during the stabilometry performed with open eyes and parallel feet recorded a worsening in all groups, Group A passes by a length equal to $1112.88 \mathrm{~mm}$ (SD 371.69) to $1404.22 \mathrm{~mm}$ (SD 564.65), Group B passes by a length equal to $1.432 .48 \mathrm{~mm}$ (SD 569.12) to a value of $1536.72 \mathrm{~mm}$ (SD 588.84), showing a deterioration of the average values ofthe sway area stabilometry (open eyes) with the use of HBP (fig 6).

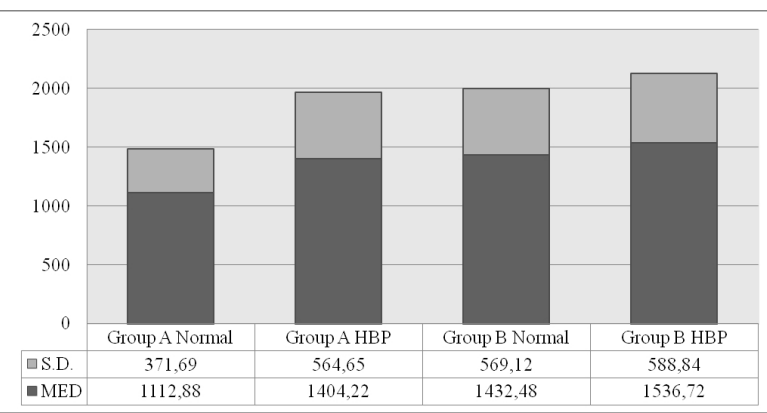

Figure 6 The difference about the sway area during the stabilometry performed with open eyes and parallel feet recorded a worsening in all groups

The difference in the length of the ball (sway area) while running the stabilometry performed with parallel feet and closed eyes recorded a worsening in all groups; group A passes from a value of $1095.48 \mathrm{~mm}$ (SD 399.85) to $1338.52 \mathrm{~mm}$ (SD 525.39), the group B passes from a length of $1453.68 \mathrm{~mm}$ (SD 616.74) to a value of $1452.56 \mathrm{~mm}$ (SD 530.62), highlighting a worsening of the average values of length of the ball in stabilometry with closed eyes using the HBP (fig 7). 


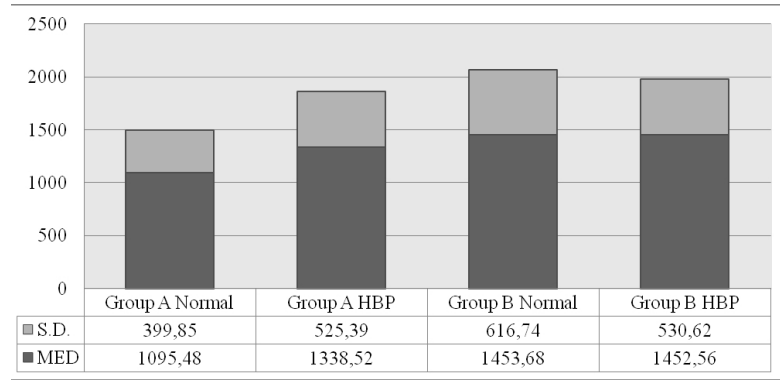

Figure 7 The difference in the length of the ball (sway area) while running the stabilometry performed with parallel feet and closed eyes recorded a worsening in all groups

The difference in the percentage of plantar support surface in static recorded a deterioration in both groups, the group A passes from a difference of $5.43 \%$ (SD 4.11) to $5.99 \%$ (SD 5.57), group B passes from a difference of $5.42 \%$ (SD 4.99) to a difference with exoskeleton amounted to $6.99 \%$ (SD 6.37), so it shows an increase of the average values of the difference percentage of the surface between right and left in static using HBP (fig 8).

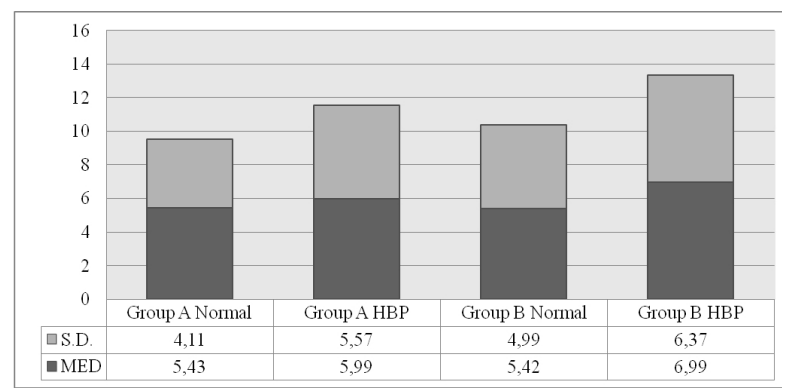

Figure 8 The difference in the percentage of plantar support surface in static recorded a deterioration in both groups

The difference of the distribution of load in percent in static analysis recorded a deterioration in both groups, the group A passes from a difference of $8.07 \%$ ( DS 6.96) to $10.57 \%$ ( DS 8.70), the group B passes from a difference equal to $11.02 \%$ (SD 8.23 ) to a difference with exoskeleton equal to 11.82 $\%$ (SD 9.04), so it shows an increase of the average values of the percentage difference of the distribution of load between right and left in static using HBP (fig 9).

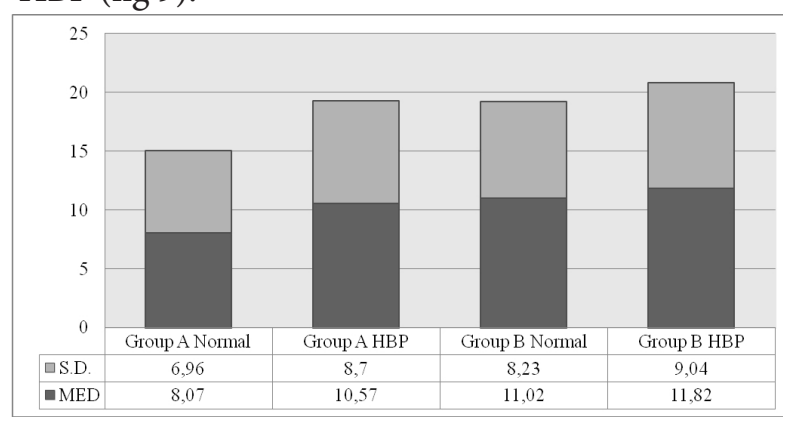

Figure 9 The difference of the distribution of load in percent in static analysis recorded a deterioration in both groups
The "coordination index" results from the evaluation of the check-up I- Moove average values with the exoskeleton HBP are higher than average values without use of HBP in both groups taken into account: in fact the group A passes by a score of $65.76 \mathrm{pt}$. (SD 18.49) without HBP to a score of 79.64 pts. (SD 10.04) with the use of the device HBP ; group B passes by a score of 70.28 pts. (SD 12.59 ) to a score of 78.96 pts. (SD 9.43) with exoskeleton (fig 10).

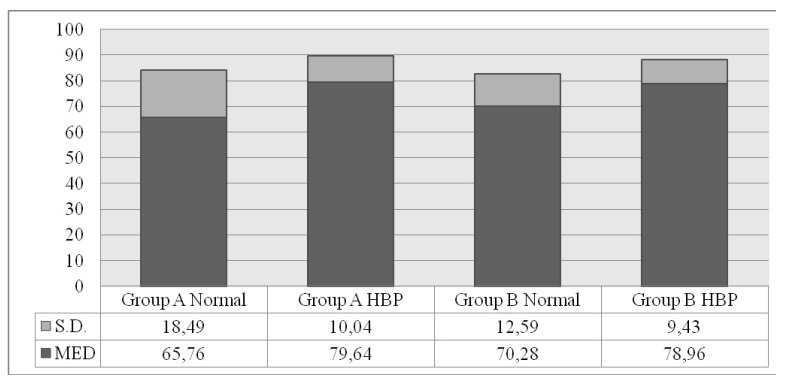

Figure 10 The "coordination index" results from the evaluation of the check-up I- Moove average values with the exoskeleton HBP

The "stability index" results from the evaluation of the check-up I- Moove average values with the exoskeleton HBP are practically unchanged comparised with the evaluation in the normal condition in both study groups, in fact the group A passes from a score of 27.36 pts. (SD 8.32) without HBP to a score of 27.84 pts. (SD 8.76) with the use of the device HBP ; in group B there is a worsening, going from a score of 28.88 pts. (SD 11.41) to a score of 28.16 pts. (SD 9.94) with exoskeleton (fig. 11)

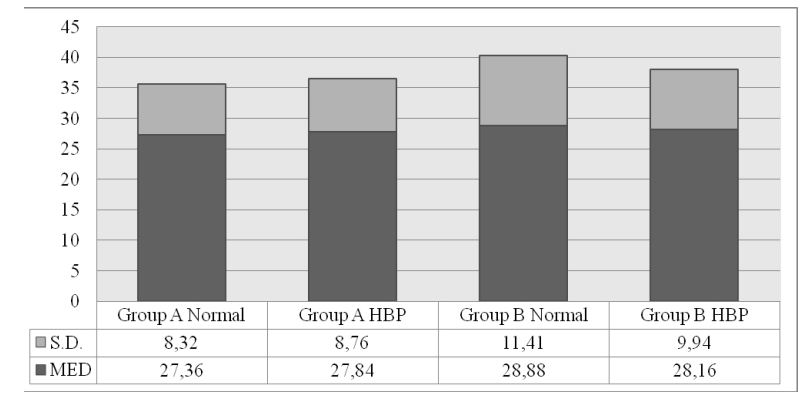

Figure 11 The "stability index" results from the evaluation of the check-up I- Moove average values with the exoskeleton HBP

In the stabilometric analysis performed with parallel feet and opened eyes the mean values of the surface of the ellipse of confidence increases with the use of HBP in both groups ;in group A switching from $18.61 \mathrm{~mm} 2$ (SD 32.81) without device to $25.25 \mathrm{~mm} 2$ (SD 40.29) with the device HBP, instead, group B is increased from a value of $20.57 \mathrm{~mm} 2$ (SD 22.51) to a value of $33.87 \mathrm{~mm} 2$ (SD 49.20 ) with the use of the exoskeleton HBP (fig 12). 


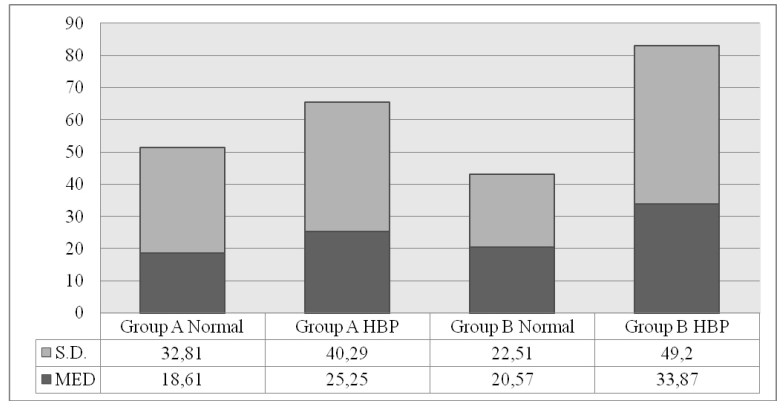

Figure 12 The stabilometric analysis performed with parallel feet and opened eyes: the mean values of the surface of the ellipse area

In the stabilometric analysis performed with parallel feet and opened eyes, the mean values of the surface of the ellipse of confidence increases with the use of HBP in a group and remains unchanged in the other; in group A, it switches from $27.24 \mathrm{~mm} 2$ (SD 41.06) without device to 27.52 $\mathrm{mm} 2$ (SD 40.01) with the device HBP ; in group B increased from a value of $18.51 \mathrm{~mm} 2$ (SD 24.14) to a value of $31.57 \mathrm{~mm} 2$ (SD 47.36) with the use of the exoskeleton HBP (fig 13).

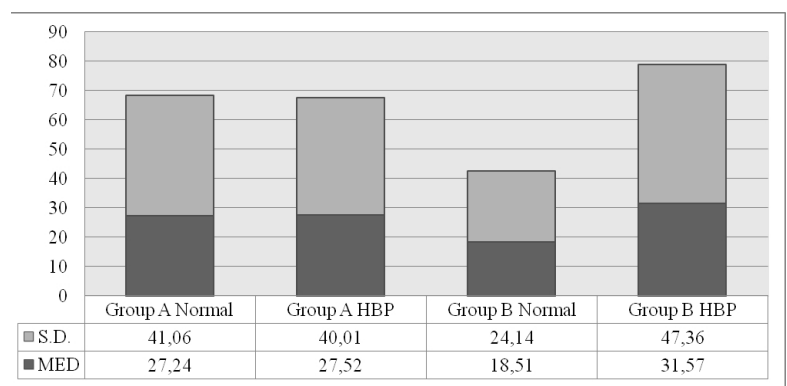

Figure 13 The stabilometric analysis performed with parallel feet and opened eyes

All patients participating in the trial have responded to an interview with questions that concerned the proposed convenience or any discomfort resulted in wearing the HBP. $60 \%$ of subjects reported that this device is comfortable, $40 \%$ of patients consider it uncomfortable (5\% coccygeal region ; $17 \%$ hip joint region ; $10 \%$ cervical region ; $3 \%$ ankle region ; $7 \%$ humeral region) (figure 11). 50 patients in the trial, the $86 \%$ of them consider the HBP exoskeleton a light weight device, on the contrary $14 \% \mathrm{f}$ them felt that it is heavy to wear (fig 14). 


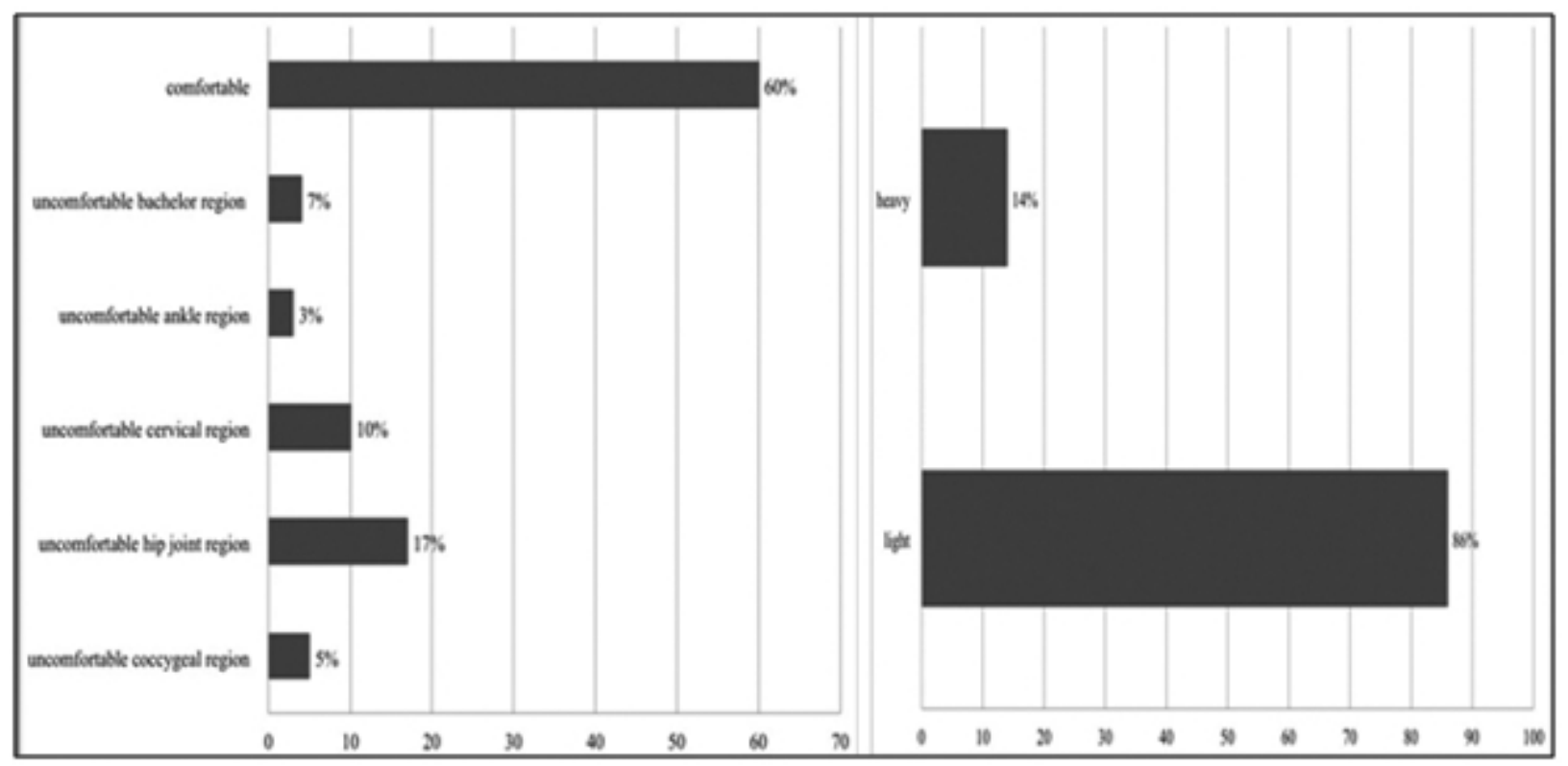

Figure 14 Questionnaire that concerned the proposed convenience or any discomfort resulted in wearing the HBP

\section{Discussion and conclusion}

Analysing these datas, it can be stated that the exoskeleton immediately changes the posture and balance of the subjects examined, surely acting also on peripheral receptors toward a physiological spinal alignment.

It was evident the ease of installation and adaptability of HBP to the patient.

At the end of the study, only partially the results set in the goals, which were a significant improvement in posture and stability, were obtained.

The cause is due to the young age and the optimum health condition of the sampled individuals and the lack of a long time of adaptation, since the evaluation procedures were carried out, for reasons related to the study, all within a single day for each patient.

An improvement of posture and a feeling of symmetry, although not statistically significant, but assessable and objectivable with the tools used in the assessment have been verified.

Being a pilot study, this research provide only evidences about the influence of HBP during its immediate use in healthy subjects.

Many studies have shown the importance of exoskeleton and robotic systems in functional and postural patients rehabilitation.[22,23,24,25]

In the paper we proposed an approach for adaptive learning of exoskeleton behaviour, on the patient's posture, motor coordination and the comfort of the HBP rehabilitative support.
The approach uses the static and dimanic stabilometry test and coordination test by I-Moove chec$\mathrm{k}$-up through the muscle activity feedback to include the human in the exoskeleton management.

Muscle activity is not an estimation of an output torque but rather an information in which direction should the torque change to minimise the human muscular effort.[26]

This essentially makes the exoskeleton torque behaviour adapt to the current human behaviour.

The proposed approach provides an interface between the human user and exoskeleton in body motion tasks in healthy subjects. We showed its feasibility on different postural tests (statics and dynamics) mechanical systems designed for trunk movements assistance. The advantage of the proposed approach is that it can operate without predetermined behaviour, it was considered comfortable by person who have worn it.

Both while using it immediately after use, the exoskeleton system(HBP) is evident the interaction with the tonic postural system and the motor coordination in the healthy subject, to the direction of rebalancing.

It dynamically forms the support and different possibility to assist the operator in the desired task.

In this study, no influence on the repeatability of the objective evaluation of tests used is shown.

To strengthen this hypothesis, further studies to determine the therapeutic effect on posture and balance of H.B.P. used both in medium and long period, through the same evaluation methods that we used in this study should be executed . 


\section{Conflict of interest}

All authors declare that there is no personal interest properties, financial, professional or other of any nature or kind in any product, service or company that could be interpreted as influencing the position presented in this manuscript.

\section{References}

1. Basmajian JV \& De Luca CJ. Muscle alive: their functions revealed by electromyography (5th ed). Baltimore; Williams and Wilkins: 1985; 19-64.

2. Tsagarakis NG, Caldwell DG. Development and Control of a Soft-Actuated Exoskeleton for Use in Physiotherapy and Training. Auton Robots. 2003;15(1):21-33. doi: 10.1023/A:1024484615192

3. Kong K, Jeon D. Design and control of an exoskeleton for the elderly and patients. Mechatronics, IEEE/ASME Transactions on. 2006;11(4):428-432.

4. Cavallaro EE, Rosen J, Perry JC, Burns S. Real-Time Myoprocessors for a Neural Controlled Powered Exoskeleton Arm. Biomedical Engineering, IEEE Transactions on. 2006;53(11):2387-2396. doi: 10.1109/ TBME.2006.880883.

5. Andreia S. P. Sousa, Augusta Silva \& João Manuel R. S. Tavares Biomechanical and neurophysiological mechanisms related to postural control and efficiency of movement: A review, Somatosensory \& Motor Research, 2012;29:4, 131-143

6. Yang D, Zhao Jd, Jiang L, Liu H. Dynamic hand motion recognition based on transient and steady-state EMG signals. International Journal of Humanoid Robotics. 2012;09(01):1250007. doi: 10.1142/S0219843612500077

7. Ijspeert AJ, Nakanishi J, Hoffmann H, Pastor P, Schaal S. Dynamical Movement Primitives: Learning Attractor Models for Motor Behaviors. Neural Computation. 2013;25(2):328373. doi: 10.1162/NECO_a_00393. pmid:23148415

8. Ijspeert AJ, Nakanishi J, Schaal S. Learning rhythmic movements by demonstration using nonlinear oscillators. In: Intelligent Robots and Systems (IROS), 2002 IEEE/RSJ International Conference on. vol. 1; 2002. p. 958-963.
9. Kandel ER, Schwartz JH, Jessell TM. Principles of Neural Science, 5th ed. New York; McGraw-Hill: 2012

10. Gams A, Ijspeert A, Schaal S, Lenarčič J. On-line learning and modulation of periodic movements with nonlinear dynamical systems. Autonomous robots. 2009;27(1):3-23. doi: 10.1007/s10514-009-9118-y.

11. Mansion J. Postural control System. Curr Opin Neurobiol. 1994 Dec;4(6):877-87.

12. Kandel ER, Schwartz JH, Jessell TM. Principles of Neural Science. 4th ed. McGraw-Hill Medical; 2000.

13. Schaal S, Atkeson CG. Constructive Incremental Learning from Only Local Information. Neural Comput. 1998;10(8):20472084. doi: 10.1162/089976698300016963. pmid:9804671

14. Yang J, Winter DA. Electromyographic amplitude normalization methods: Improving their sensitivity as diagnostic tools in gait analysis. Arch Phys Med Rehabil. 1984;65:517-521. pmid:6477083

15. De Luca CJ. The use of surface electromyography in biomechanics. Journal of Applied Biomechanics. 1997;13(2):135-163. doi: 10.1016/0021-9290(94)91124-x

16. De Luca CJ, Gilmore LD, Kuznetsov M, Roy SH. Filtering the surface EMG signal: Movement artifact and baseline noise contamination. Journal of Biomechanics. 2010;43(8):15731579. doi: 10.1016/j.jbiomech.2010.01.027. pmid:20206934.

17. Di Russo F, Berchicci M, Perri RL, Ripani FR, Ripani M., Avenanti A: A Passive Exoskeleton Can Push Your Life Up: Application on Multiple Sclerosis Patients., ed. PLoS ONE. 2013;8(10):e77348. doi:10.1371/journal. pone. 0077348.

18. Gianturco V, Troisi G, Ripani M, Marigliano V. Impact of exoskeleton Human Body Posturizer on the fall risk in the elderly: A randomized controlled trial. European geriatric medicine :s45-s46. doi: 10.1016/j.eurger.2013.07.149.

19. Zoss AB, Kazerooni H, Chu A. Biomechanical design of the Berkeley lower extremity exoskeleton (BLEEX). Mechatronics, IEEE/ ASME Transactions on. 2006;11(2):128-138. doi: 10.1109/TMECH.2006.871087 
20. Solovykh EA, Maksimovskaya LN, Bugrovetskaya OG, Bugrovetskaya EA. Comparative analysis of methods for evaluation of stabilometry parameters. Bull Exp Biol Med. 2011 Dec;152(2):266-72

21. Burgar CG, Lum PS, Shor PC, Machiel Van der Loos HF. Development of robots for rehabilitation therapy: the Palo Alto VA/Stanford experience. J Rehabil Res Dev. 2000; 37(6):663-73.

22. Hesse S, Schulte-Tigges G, Konrad M, Bardeleben A, Werner C. Robot-assisted arm trainer for the passive and active practice of bilateral forearm and wrist movements in hemiparetic subjects. Arch Phys Med Rehabil. 2003;84(6): 915-20.
23. Volpe BT, Krebs HI, Hogan N, Edelstein OTR L, Diels C, Aisen M. A novel approach to stroke rehabilitation: robot-aided sensorimotor stimulation. Neurology. 2000;54(10): 1938-44

24. Fasoli SE, Krebs HI, Stein J, Frontera WR, Hogan N. Effects of robotic therapy on motor impairment and recovery in chronic stroke. Arch Phys Med Rehabil. 2003; 84(4):477-82.

25. Peternel L, Noda T, Petrič T, Ude A, Morimoto J, Babič J Adaptive Control of Exoskeleton Robots for Periodic Assistive Behaviours Based on EMG Feedback Minimisation.PLoS One. 2016 Feb 16;11(2):e0148942. doi: 10.1371/journal.pone.0148942. eCollection 2016. 